\title{
Radio spectral index variations of the SNR HB3
}

\author{
W. W. Tian ${ }^{1,2}$ and D. Leahy ${ }^{2}$ \\ ${ }^{1}$ National Astronomical Observatories, CAS, Beijing 100012, PR China \\ e-mail: tww@ns.bao.ac.cn \\ 2 Department of Physics \& Astronomy, University of Calgary, Calgary, Alberta T2N 1N4, Canada \\ Received 24 November 2004 / Accepted 26 January 2005
}

\begin{abstract}
We present images of the SNR HB3 at both $408 \mathrm{MHz}$ and $1420 \mathrm{MHz}$ from the Canadian Galactic Plane Survey mainly based on data from the Synthesis Telescope of the Dominion Radio Astrophysical Observatory. We reproduce the $100 \mathrm{~m}$-Effelsberg HB3 image at $2695 \mathrm{MHz}$ at large scale, and find that there exists a background emission gradient across the HB3 area. Based on our analysis of background emission and the boundary between W3 and HB3, we give HB3's flux density as $68.6 \pm 11.5 \mathrm{Jy}$ at $408 \mathrm{MHz}$ and $44.8 \pm 12.0 \mathrm{Jy}$ at $1420 \mathrm{MHz}$, after subtracting flux from compact sources within HB3. The integrated flux-density-based spectral index between $408 \mathrm{MHz}$ and $1420 \mathrm{MHz}$ is $0.34 \pm 0.15$. The averaged T-T plot spectral index using all subareas is 0.36 . Our measurement values are less than a previously published value of 0.6 . The $408-1420 \mathrm{MHz}$ spectral index varies spatially in HB3 in the range 0.1 to 0.7 . We investigate the data used by previous authors, and consider more data at $232 \mathrm{MHz}, 3650 \mathrm{MHz}$ and $3900 \mathrm{MHz}$ which are not included in previous calculations. There is evidence for two spectral indices for HB3 in the radio band, i.e., $0.63(38-610 \mathrm{MHz})$ and $0.32(408-3900 \mathrm{MHz})$. This is consistent with the spatial variations: the low frequency data mainly reflects the steeper indices and the high frequency data mainly reflects the flatter indices.
\end{abstract}

Key words. ISM: individual: HB3 - radio continuum: ISM - ISM: supernova remnants

\section{Introduction}

The evolved supernova remnant (SNR) HB3 (G132.7+1.3; Landecker et al. 1987), as one of the largest galactic SNRs currently known, has been previously observed by Caswell (1967) and Landecker et al. (1987) in the radio continuum, by Leahy et al. (1985) in X-rays, by Routledge et al. (1991) in the HI-line, by Fesen et al. (1995) in H $\alpha$, and by Koralesky et al. (1998) in hydroxy $1(\mathrm{OH})$. Its known basic physical features are $60 \times 80 \mathrm{pc}$ in size based on a distance of $2 \mathrm{kpc}$ (Routledge 1991). It interacts with the molecular gas in which $\mathrm{W} 3$ is embedded, but there is no evidence for a direct interaction of W3 and HB3; strong radio-optical correlation is also observed. There is no detailed study on HB3's radio spectrum so far. The complex surrounding of the SNR embedded in a star-forming region that contains the W3 HII complex makes it difficult for previous authors to spatially separate HB3 from the W3 complex and to estimate the background emission level in order to obtain an accurate integrated flux density of HB3. Many previous radio observational data on HB3 are of limited use due to the limited spatial resolution. Although a spectrum index for HB3 of about 0.6 has been suggested, it is worth further study. Therefore, we use new HB3 data from the recently finished Canadian Galactic Plane Survey, described in Sect. 2, and present the results and a discussion of HB3's spectrum in Sect. 3. A summary of findings is given in Sect. 4.

\section{Observations and image analysis}

The $408 \mathrm{MHz}$ and $1420 \mathrm{MHz}$ data sets come from the Canadian Galactic Plane Survey (CGPS) which is described in detail by Taylor et al. (2003). The radio continuum observations' resolution is below $1^{\prime} \times 1^{\prime} \operatorname{cosec}(\delta)$ at $1420 \mathrm{MHz}$ and $3.4^{\prime} \times 3.4^{\prime} \operatorname{cosec}(\delta)$ at $408 \mathrm{MHz}$, which is the highest available resolution for HB3 so far. Because Dominion Radio Astrophysical Observatory (DRAO) Synthesis Telescope (ST) observations in the CGPS are generally not sensitive to structures larger than an angular size scale of about $3.3^{\circ}$ at $408 \mathrm{MH}$ and $56^{\prime}$ at $1420 \mathrm{MHz}$, the CGPS includes data from the $408 \mathrm{MHz}$ all-sky survey of Haslam et al. (1982) which has an effective resolution of $51^{\prime}$ and the Effelsberg $1.4 \mathrm{GHz}$ Galactic plane survey of Reich et al. (1990, 1997) with resolution $9.4^{\prime}$ for large scale emission (the single-dish data are freely available by http://www. mpifr-bonn.mpg.de/survey.html). Taylor et al. (2003) mentioned artifacts of the CGPS images arising from the single- antenna data. We check the HB3 map from the 408 $\mathrm{MHz}$ all-sky survey, and confirm that there exists a type of artifact, i.e. low-level striping that appears as discontinuities across lines of constant Right Ascension. The maximum amplitude of the striping with scale of $3^{\circ}$ is less than $6 \%$. In the $408 \mathrm{MHz}$ single-antenna map, HB3 is located between two adjacent strips, and is very little contaminated by the artifact, i.e, only part of B1 region of HB3 (see Fig. 1) is influenced by the 

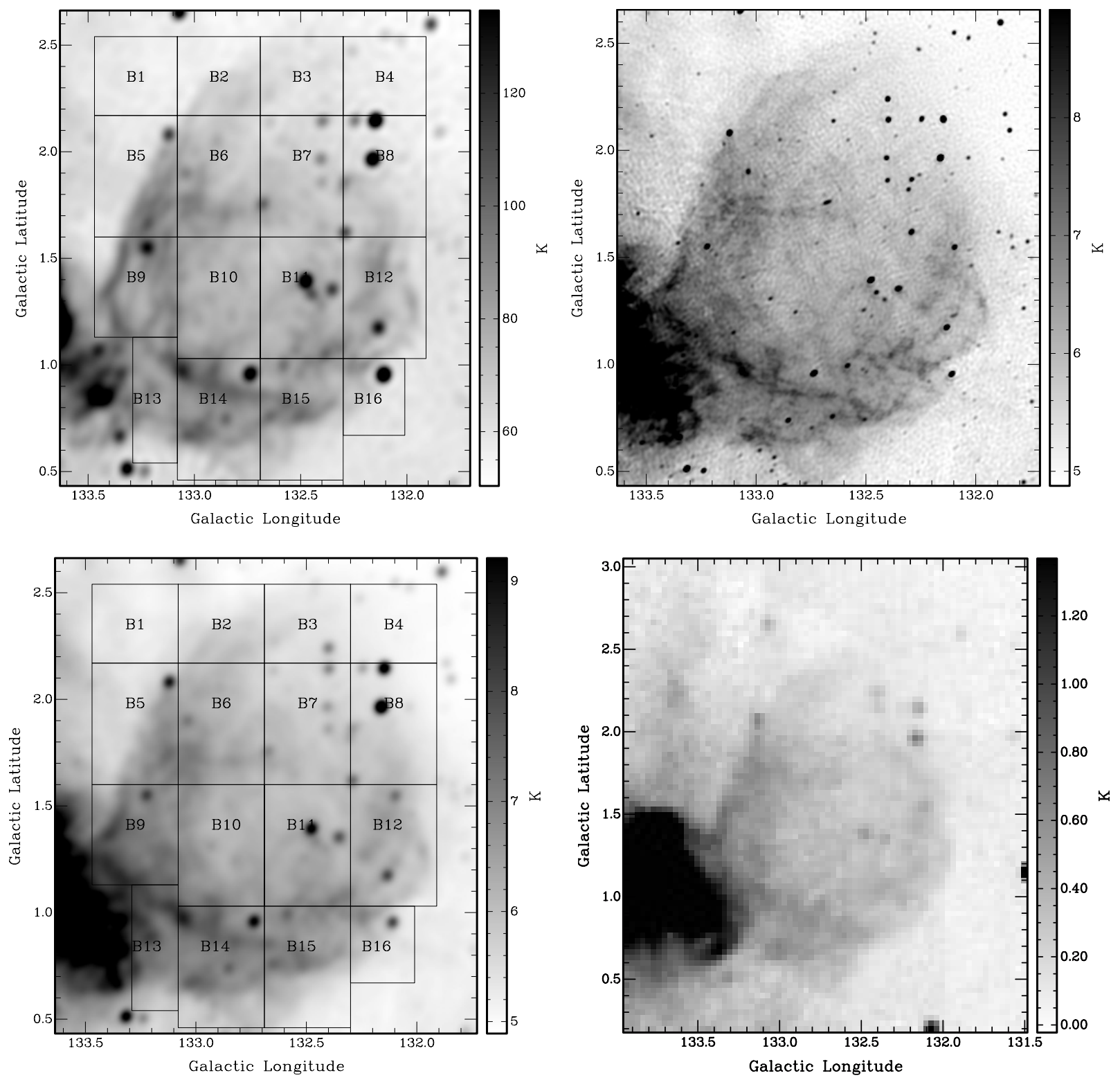

Fig. 1. The first row shows the HB3 CGPS maps at $408 \mathrm{MHz}$ (left) and $1420 \mathrm{MHz}$ (right). The left panel of the second row shows the $1420 \mathrm{MHz}$ map convolved to the same resolution as the $408 \mathrm{MHz}$ map. The $2695 \mathrm{MHz}$ Bonn map is shown at right in the second row. The 16 boxes used for T-T plots are shown in the upper and lower left panels.

artifact. In the $1420 \mathrm{MHz}$ Effelsberg map of the HB3, sidelobes have positions to the east and west from the origin. However the amplitude of the effect is less than $2 \%$ of the brightness of HB3 so it is not very significant. Since the CGPS integrates both single-antenna and interferometer data, information on HB3's structure on all angular scales larger than the resolution limit is included. By using a special image-processing procedure, the dynamic range of the continuum images reaches up to 10000 at $1420 \mathrm{MHz}$ and up to 5000 at $408 \mathrm{MHz}$, yielding essentially noise-limited images with an rms of $0.3 \mathrm{mJy}^{\text {beam }}{ }^{-1}$ at $1420 \mathrm{MHz}$ and $3 \mathrm{mJy}_{\text {beam }}^{-1}$ at $408 \mathrm{MHz}$ (Taylor et al. 2003).

Estimating HB3's flux density at each frequency is the first important step to study its spectrum variation. We analyze the HB3 images using the DRAO export software package to get HB3's flux density at each frequency. To study thermal contamination from $\mathrm{W} 3$ and keep it as small as possible, we study the radio spectrum in 16 different regions in HB3. The influence of compact sources within HB3 is very much reduced by two alternate methods: a) compact sources within HB3 are each fit by Gaussian components plus a twisted plane background, then the source component is removed from the image; b) a small area including each compact source is completely removed from the analysis. Our analysis shows that the latter method reduces the effect of compact sources on the spectral index of HB3 to minimum.

\section{Results and discussions}

\section{1. $\mathrm{HB} 3$ structure at $408 \mathrm{MHz}$ and $1420 \mathrm{MHz}$}

The CGPS maps at $408 \mathrm{MHz}$ and $1420 \mathrm{MHz}$ are shown in the upper left and right panels of Fig. 1. The lower left panel 

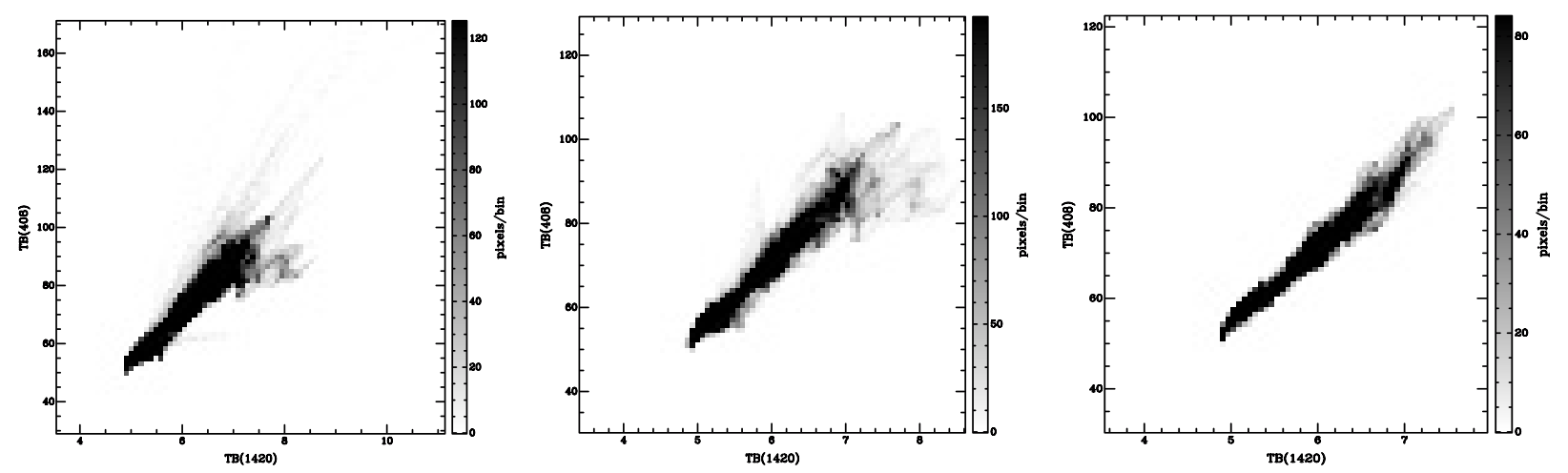

Fig. 2. $408 \mathrm{MHz}-1420 \mathrm{MHz}$ T-T plots using one box covering HB3, from left to right: plot for map including compact sources $(\alpha=0.25 \pm 0.12)$; plot for map with Gaussian fits to compact sources subtracted $(\alpha=0.19 \pm 0.11)$; plot for regions including compact sources removed from analysis $(\alpha=0.22 \pm 0.06)$.

shows the $1420 \mathrm{MHz}$ map which has been convolved to the same resolution as the $408 \mathrm{MHz}$ map prior to source removal. For reference we also reproduce the $2695 \mathrm{MHz}$ Effelsberg map (lower right; Fürst et al. 1990) in Fig. 1. The Effelsberg map has an resolution of $4.3^{\prime}$ and a sensitivity of $50 \mathrm{mKT} T_{\mathrm{B}}$. The $408 \mathrm{MHz}$ CGPS image is generally similar to previously published $408 \mathrm{MHz}$ images (Landecker et al. 1987; Fesen et al. 1995), but shows significantly more detail and clearer filament features, as well as greatly reduced artifacts due to compact sources and W3. This is due to the higher sensitivity and dynamic range of the CGPS data. Our $1420 \mathrm{MHz}$ map shows rich weak emission features with much better quality than the Fesen et al. (1995) map. The CGPS maps at both frequencies are free from any strong artifacts caused by W3 or due to strong compact sources, which appear in the Fesen et al. maps. The compact sources within HB3 are well resolved at both frequencies. The higher spatial resolution at $1420 \mathrm{MHz}$ means more compact sources are seen. Both $1420 \mathrm{MHz}$ and $408 \mathrm{MHz}$ CGPS maps have sufficient spatial resolution to identify and correct for all compact sources that have significant flux.

\subsection{HB3 spectral index}

HB3's 408-1420 MHz spectral index $\alpha\left(S_{v}=S_{0} v^{-\alpha}\right)$ is either about 0.34 based on our direct calculations by using integrated flux densities at both frequencies or 0.36 by the T-T plot method. The principle of the $\mathrm{T}-\mathrm{T}$ plot method is that brightness temperature spectral indices $\left(T_{v}=T_{0} v^{-\beta}\right)$ are calculated by fitting a linear relation to the $T_{1}-T_{2}$ values of all pixels within a given map region. Here $T_{1}$ is the brightness temperature of a given map pixel at the first frequency. $T_{2}$ is the brightness temperature of the same pixel at the second frequency. The brightness temperature spectral index $\beta$ is then derived from the slope of the curve. The flux density spectral index $\alpha$ is related to the brightness temperature spectral index $\beta$ by $\beta=\alpha+2$. We use spectral index to refer to the flux density spectral index $\alpha$ in this paper unless we specifically say otherwise. The T-T plot method has widely been accepted and used for spectral index calculation (e.g., Zhang et al. 1997; Leahy \& Roger 1998).

In Leahy \& Roger (1998), the effect of compact sources is reduced by avoiding including any strong sources in the regions analyzed. For the T-T plot spectral index, we choose a single analysis box covering $131.935^{\circ}$ to $133.285^{\circ}$ Galactic longitude, and $0.725^{\circ}$ to $2.705^{\circ}$ Galactic latitude. This box is conservatively chosen to include most of HB3 yet have its leftmost boundary clearly right of the edge of W3. This region yields the T-T plots shown in Fig. 2 and the spectral index for the three cases: including, subtracting and excluding compact sources within HB3 (see Fig. 2 caption for details). Since the brightest compact sources have a steeper spectrum than HB3, they show up in the T-T plot as the steeper lines of points (Fig. 2, left panel). The middle panel is the T-T plot from the map with compact sources subtracted by subtraction of the Gaussian components fit to the original map. In both the left and middle panels, there is a significant amount of emission to the right of the main emission band from HB3. This emission has an irregular structure which has no simple interpretation in terms of a spectral index. This irregular structure could be due to weak artifacts from the compact sources, which are present before and after Gaussian component subtraction. The second method we used to remove compact sources is to remove a small region including the compact source from the analysis. The region is taken to be a few beamwidths wide, so that any contribution from the compact source is below $1 \%$ of the diffuse SNR emission. Thus when a compact source is removed, any artifacts associated with the compact source are also removed. This is proven by the right plot of Fig. 2, where the compact sources have been removed and the T-T plot does not show the irregular structure.

In view of a likely gradient of the thermal emission due to W3, we consider the effect of such a gradient on the T-T plot spectral index. For a large area such as the box for the whole of HB3 the thermal emission component has a significant variation which is larger at $1420 \mathrm{MHz}$ than $408 \mathrm{MHz}$. When this is added to the non-thermal emission and a T-T plot is made, the set of points is broadened in the direction parallel to the $T_{B}(1420)$ axis more than in the direction parallel to the $T_{B}(408)$ axis. The broadening is equal to the difference in brightness temperature due to the thermal component across the map at each frequency. If we choose smaller boxes, e.g. $1 / 4$ size in linear dimension, the broadening due to difference in thermal emission across the box is reduced by a factor 
Table 1. HB3 408-1420 MHz spectral index for regions B1 to B16.

\begin{tabular}{cccc}
\hline \hline Sp. index & $\alpha$ & $\alpha$ & $\alpha$ \\
\hline Area No. & Including compact sources & Compact sources subtracted & Compact sources removed \\
\hline B1 & $0.06 \pm 0.50$ & $0.03 \pm 0.73$ & $0.07 \pm 0.49$ \\
B2 & $0.29 \pm 0.07$ & $0.29 \pm 0.07$ & $0.29 \pm 0.07$ \\
B3 & $0.12 \pm 0.21$ & $0.18 \pm 0.23$ & $0.18 \pm 0.14$ \\
B4 & $0.46 \pm 0.09$ & $0.30 \pm 0.20$ & $0.28 \pm 0.14$ \\
B5 & $0.51 \pm 0.06$ & $0.54 \pm 0.06$ & $0.55 \pm 0.05$ \\
B6 & $0.43 \pm 0.06$ & $0.46 \pm 0.08$ & $0.45 \pm 0.08$ \\
B7 & $0.52 \pm 0.05$ & $0.43 \pm 0.07$ & $0.31 \pm 0.09$ \\
B8 & $0.45 \pm 0.04$ & $0.31 \pm 0.05$ & $0.25 \pm 0.02$ \\
B9 & $0.12 \pm 4.15$ & $0.05 \pm 4.10$ & $0.11 \pm 0.79$ \\
B10 & $0.12 \pm 0.10$ & $0.09 \pm 0.10$ & $0.09 \pm 0.10$ \\
B11 & $0.60 \pm 0.12$ & $0.39 \pm 0.12$ & $0.40 \pm 0.07$ \\
B12 & $0.45 \pm 0.06$ & $0.41 \pm 0.07$ & $0.36 \pm 0.04$ \\
B13 & $0.10 \pm 0.14$ & $0.07 \pm 0.13$ & $0.05 \pm 0.13$ \\
B14 & $0.45 \pm 0.02$ & $0.38 \pm 0.04$ & $0.45 \pm 0.01$ \\
B15 & $0.29 \pm 0.02$ & $0.32 \pm 0.03$ & $0.29 \pm 0.02$ \\
B16 & $0.25 \pm 0.03$ & $0.25 \pm 0.03$ & $0.23 \pm 0.02$ \\
\hline
\end{tabular}

of 4. Thus we consider smaller boxes to reduce the effect of a thermal emission gradient. There is a limit on how small one goes, as the box needs to be large enough to contain significant variation in the non-thermal emission.

Thus we divide HB3 into the 16 areas shown in Fig. 1. The areas were chosen to cover HB3 completely, then the regions B9 and B13 were adjusted to include the leftmost part of HB3 while avoiding emission from $\mathrm{W} 3$ as best as possible. For each region the spectral index was obtained between $408 \mathrm{MHz}$ and $1420 \mathrm{MHz}$ by the T-T plot method. Table 1 lists the results for the three cases of analysis: including compact sources, subtracting compact sources, and removing compact sources. There is not a large difference in results between the three methods, however visual inspection of the T-T plots shows that the third method produces the most reliable results, as it did for the large single box covering HB3 shown in Fig. 2. We mention that compact sources' influence on the spectral index calculation is obvious in some areas such as boxes $4,7,8$ and 11 . Thus from now on we discuss spectral indices derived with compact sources removed, unless specified otherwise. The spectral index of Box 1 is very flat with a large error. As we mentioned in Sect. 2, Box 1 is influenced by the artifact arising from the $408 \mathrm{MHz}$ single antenna data, and in fact is mostly outside the HB3 emission area; this is a measure of the thermal background emission. The derived spectral index values are between 0.2 to 0.5 except those for Boxes 9, 10 and 13. The averaged value of spectral index excluding large error regions B1 and $\mathrm{B} 9$ is $0.30 \pm 0.07$. This is larger than the value of the whole of HB3 of $0.22 \pm 0.06$ (see caption of Fig. 2), consistent with a reduced contribution from the thermal gradient.

Because the T-T plots of boxes 2, 5, 6, 9, 10, 13 show a complex distribution indicative of multiple spectral indices, we divide each of them into four equal-size subareas: a) upper left; b) upper right; c) lower left; and d) lower right. In many cases the subdivision separates areas of different spectral index so that the T-T plot method can yield a useful spectral index. Table 2 gives the spectral index of each subarea obtained by
Table 2. HB3 408-1420 MHz spectral index for subareas.

\begin{tabular}{cccc}
\hline \hline Sp. index & \multicolumn{2}{c}{$\alpha$} & $\alpha$ \\
\hline Area No. & Automatic fit & Manual fit & Box average \\
\hline SUBBOX 2a & $-0.2 \pm 4.0$ & 0.3 & 0.35 \\
SUBBOX 2b & $0.3 \pm 0.03$ & 0.4 & \\
SUBBOX 2c & $0.6 \pm 0.1$ & $0.4,0.6$ & \\
SUBBOX 2d & $0.1 \pm 0.2$ & $0.0,0.4$ & \\
\hline SUBBOX 5a & $0.4 \pm 0.3$ & $0.2,0.3$ & 0.49 \\
SUBBOX 5b & $0.4 \pm 0.02$ & $0.1,0.7$ & \\
SUBBOX 5c & $0.6 \pm 0.2$ & $0.6,0.7$ & \\
SUBBOX 5d & $0.5 \pm 0.4$ & $0.6,0.7$ & \\
\hline SUBBOX 6a & $0.5 \pm 0.1$ & 0.4 & 0.50 \\
SUBBOX 6b & $0.0 \pm 0.1$ & $0.1,0.6$ & \\
SUBBOX 6c & $-0.1 \pm 0.7$ & 0.6 & \\
SUBBOX 6d & $0.8 \pm 0.1$ & $0.6,0.7$ & \\
\hline SUBBOX 9a & $0.6 \pm 0.2$ & $0.6,0.9$ & 0.45 \\
SUBBOX 9c & $0.0 \pm 4.1$ & $0.3,0.4$ & \\
SUBBOX 9d & $-0.1 \pm 4.2$ & $0.2,0.3$ & \\
\hline SUBBOX 10a & $0.1 \pm 0.2$ & 0.4 & 0.51 \\
SUBBOX 10b & $0.3 \pm 4.3$ & $0.5,0.7$ & \\
SUBBOX 10c & $0.3 \pm 0.1$ & $0.5,0.7$ & \\
SUBBOX 10d & $0.3 \pm 0.2$ & $0.3,0.6$ & \\
\hline SUBBOX 13a & $-0.9 \pm 4.6$ & $0.2,0.5,0.7$ & 0.33 \\
SUBBOX 13b & $-0.5 \pm 0.7$ & $-0.1,0.0,0.4$ & \\
SUBBOX 13c & $-0.1 \pm 0.5$ & $0.2,0.5$ & \\
SUBBOX 13d & $0.1 \pm 0.6$ & $0.1,0.7$ & \\
\hline
\end{tabular}

the automated linear fit to the T-T plot and by a manual linear fit in Cols. 2 and 3, resp., and the average value of the manual linear fits for each box in the final column. When there are multiple lines of points in the T-T plots, the manual linear fits give a more reliable spectral index. The values in Table 2 reveal that the spectrum index can be different in different subareas or even vary within a single subarea. The average values for B2, B5 and B6 is similar to those given in Table 1, but for B9, B10 and B13 the values from the more detailed analysis differ significantly from that from the automatic linear fit. This is due to 
Table 3. Integrated flux and spectral index of the whole area and five subareas* of HB3 and of Compact Sources (CSs) at $1420 \mathrm{MHz}$ and $408 \mathrm{MHz}$.

\begin{tabular}{ccccccccc}
\hline \hline Freq. & CSs & HB3 area & HB3+CSs & HB3 Sub-1 & HB3 Sub-2 & HB3 Sub-3 & HB3 Sub-4 & HB3 Sub-5 \\
\hline MHz & Jy & Jy & Jy & Jy & Jy & Jy & Jy & Jy \\
\hline 408 & $5.3 \pm 0.4$ & $68.6 \pm 11.5$ & $73.9 \pm 11.9$ & $41.4 \pm 7.9$ & $12.5 \pm 0.9$ & $4.1 \pm 0.1$ & $13.6 \pm 0.9$ & $1.3 \pm 0.2$ \\
1420 & $2.4 \pm 0.2$ & $44.8 \pm 12.0$ & $47.2 \pm 12.2$ & $23.2 \pm 3.8$ & $8.3 \pm 1.3$ & $3.6 \pm 0.2$ & $10.0 \pm 1.7$ & $0.8 \pm 0.1$ \\
$\alpha$ & $0.64 \pm 0.10$ & $0.34 \pm 0.15$ & $0.36 \pm 0.15$ & $0.47 \pm 0.16$ & $0.33 \pm 0.07$ & $0.11 \pm 0.01$ & $0.34 \pm 0.06$ & $0.34 \pm 0.08$ \\
\hline
\end{tabular}

* Subarea 1 is sum of B2, B3, B4, B6, B7, B8, B10, B11 and B12; Subarea 2 is sum of B1, B5 and B9; Subarea 3 is B13; Subarea 4 is sum of B14 and B15; Subarea 5 is B16.

the error in fitting a mixture of lines of data points with a single model line. We note that B9 and B13 are the two boxes closest to W3 and when we fit the subboxes using manual linear fits, the correct $\mathrm{T}-\mathrm{T}$ plot spectral indices are 0.45 and 0.33 . These values are consistent with spectral indices for the other regions of HB3. The average spectral index of the whole of HB3 (excluding region B1) using areas and subareas is 0.36 . This is the best value we can get using the T-T plot method.

Next, in order to continue analyzing the thermal background influence on HB3 in detail, we reproduce the $2695 \mathrm{MHz}$ Effelsberg HB3 map in Fig. 1. The map shows there generally exists a uniform thermal background emission gradient from $400 \mathrm{mK}$ nearby the border between W3 and HB3 to about $80 \mathrm{mk}$ at HB3's northwestern edge. This gradient is also seen at a lower level in the $1420 \mathrm{MHz}$ map. Considering the general background emission gradient, we calculate the integrated flux density of HB3. With background values averaged from around the whole boundary of HB3, the integrated flux densities are $68.6 \pm 11.5 \mathrm{Jy}$ at $408 \mathrm{MHz}$ and $44.8 \pm 12.0 \mathrm{Jy}$ at $1420 \mathrm{MHz}$ (see Table 3). This choice of background subtracts any smooth gradient of background emission from HB3. Here we have used the compact source removed maps at both $408 \mathrm{MHz}$ and $1420 \mathrm{MHz}$ and also corrected for the area of HB3 removed in the compact source removal. The background uncertainty and the calibration uncertainty both contribute significantly $(\sim 10 \%)$ to the uncertainty in integrated flux.

The resulting spectral index is $0.34 \pm 0.15$ between $408 \mathrm{MHz}$ and $1420 \mathrm{MHz}$. Table 3 gives fluxes and spectral indices for HB3 as a whole and for five subareas with background calculated separately for each subarea. The subareas have compact sources removed (the subarea definition is given in Table 3). For comparison, fluxes and spectral indices for the whole of HB3 are given including compact sources as well for compact sources removed, and the sum of fluxes of compact sources within HB3 is given. The results show that compact sources contribute less than 7\% to HB3's flux and only have a small effect on the spectral index. Also, except for subarea 3, the subareas' $\alpha$ values are consistent with the spectral index $0.34 \pm 0.15$ for the whole of HB3. Subarea 3 is the region most likely contaminated by additional non-smooth thermal emission from $\mathrm{W} 3$.

Finally, we collect published integrated flux densities and errors for HB3 at other frequencies in Table 4 and show them in Fig. 3. A new HB3 integrated flux density $34.7 \pm 12.9$ at $2695 \mathrm{MHz}$ is obtained by summing values of the integrated fluxes of the five subareas defined in Table 3. We have
Table 4. Published integrated flux densities of HB3.

\begin{tabular}{cccc}
\hline \hline $\begin{array}{c}\text { Freq. } \\
\text { MHz }\end{array}$ & $\begin{array}{c}\text { Beamwidth } \\
\text { arcmin }\end{array}$ & $\begin{array}{c}\text { Flux den. } \\
\text { Jy }\end{array}$ & References \\
\hline 38.0 & $45 \times 45$ & $380.0 \pm 80$. & Caswell (1967) \\
83.0 & $59 \times 31$ & $180.0 \pm 30$. & Kovalenko et al. (1994) \\
102.0 & $48 \times 25$ & $165.0 \pm 30$. & Kovalenko et al. (1994) \\
111.0 & $44 \times 23$ & $155.0 \pm 30$. & Kovalenko et al. (1994) \\
178.0 & $23 \times 19$ & $120.0 \pm 20$. & Caswell (1967) \\
232.0 & $3.8 \times 4.3$ & $120.0 \pm 20$ & Zhu (1993) \\
408.0 & $3.5 \times 4.0$ & $75.0 \pm 15$. & Landecker et al. (1987) \\
408.0 & $3.4 \times 3.8$ & $68.6 \pm 11.5$ & This paper \\
610.0 & $16 \times 20$ & $60.0 \pm 18$. & Landecker et al. (1987) \\
863.0 & $14.6 \times 14.3$ & $51.5 \pm 3.5$ & Reich et al. (2003) \\
1420.4 & $1 \times 1.1$ & $44.8 \pm 12.0$ & This paper \\
2695.0 & $5 \times 5$ & $34.0 \pm 7.0$ & Velusamy \& Kundu (1974) \\
2695.0 & $3.4 \times 3.4$ & $34.7 \pm 12.9$ & This paper \\
3650.0 & $1 \times 10$ & $35.0 \pm 10.5$ & Trushkin (1987) \\
3900.0 & $1 \times 10$ & $33.0 \pm 9.9$ & Trushkin (1987) \\
\hline
\end{tabular}

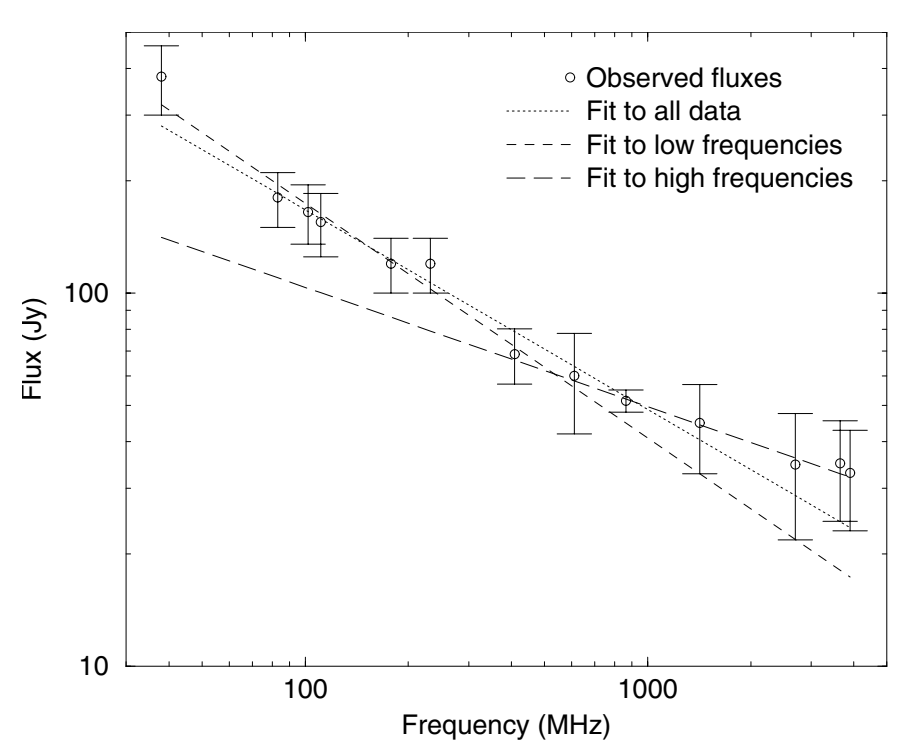

Fig. 3. Radio spectrum of HB3. The fit to all data has $\alpha=0.54$; the fit to data between $38 \mathrm{MHz}$ and $610 \mathrm{MHz}$ has $\alpha=0.63$; and the fit to data between $408 \mathrm{MHz}$ and $3900 \mathrm{MHz}$ has $\alpha=0.32$.

extrapolated total compact source fluxes to these other frequencies, using the $408-1420 \mathrm{MHz}$ spectral index upper and lower limits. The compact sources contribution is highest at $38 \mathrm{MHz}$ at $\sim 9 \%$. It smoothly decreases to $\sim 4 \%$ at $3900 \mathrm{MHz}$. In the fitting below, we omit Trushkin et al. (1987)'s measurement of $70 \pm 5.0 \mathrm{Jy}$ at $960 \mathrm{MHz}$. In fact, Trushkin himself (2002) 
predicted that it should be about $48 \mathrm{Jy}$ at $960 \mathrm{MHz}$ in his spectrum calculation of HB3, i.e, about $30 \%$ of the measurement value is discarded. Considering Trushkin et al.'s observations at $3900 \mathrm{MHz}$ and $3650 \mathrm{MHz}$ have a large elliptical beam and suffer potentially from thermal contamination from the W3 HII complex, we also use $30 \%$ of their measurement as the error of each value. Since in all cases the errors are dominated by systematic errors and are estimates by the authors without details given it is difficult to interpret them. Here we take the published errors as 1- $\sigma$ errors but if they are interpreted as 2- $\sigma$ errors, all of the $\chi^{2}$ values increase by a factor of 4 . The best-fit leastsquares fit for a single power-law has $\alpha=0.54$ and $\chi^{2}$ of 5.3 for 11 degrees of freedom (d.o.f.). Two power-laws results in significantly reduced $\chi^{2}$ : from $38 \mathrm{MHz}$ to $610 \mathrm{MHz}$, the bestfit $\alpha$ is 0.63 with $\chi^{2}$ of 1.9 for 6 d.o.f., and from $408 \mathrm{MHz}$ to $3900 \mathrm{MHz}$ the best-fit is $\alpha$ is 0.32 with $\chi^{2}$ of 0.2 for 5 d.o.f. Figure 3 shows the three power-laws. Here we conclude that the fit with two power-laws better fits the data, but the significance of the improvement is difficult to assess due to the difficulty in assessing the systematic errors in the data.

\subsection{Comparison with previously published integrated fluxes}

Landecker et al. (1987) presented DRAO ST observations on $\mathrm{HB} 3$ at $408 \mathrm{MHz}$, and estimated its flux density $75 \pm 15 \mathrm{Jy}$ at $408 \mathrm{MHz}$ after they considered that the plateau of emission around W3 and the bright ridge near the apparent perimeter of HB3 are thermal and separated them from HB3. Landecker et al. considered the location border between W3 and HB3. Their work is confirmed and refined by our work based on the current higher dynamic range data. We obtained maps including W3 and HB3 at $327 \mathrm{MHz}$ from WENSS (resolution $54^{\prime \prime} \times 54^{\prime \prime} \operatorname{cosec}(\delta)$; Rengelink et al. 1997), $408 \mathrm{MHz}$ and $1420 \mathrm{MHz}$ from DRAO (resolution $3.4^{\prime} \times 3.4^{\prime} \operatorname{cosec}(\delta)$, and $1^{\prime} \times 1^{\prime} \operatorname{cosec}(\delta)$, respectively), $2695 \mathrm{MHz}$ from the Effelsberg 100-m (resolution 4.3'; Fürst et al. 1990) and $4850 \mathrm{MHz}$ from GB6 (resolution 3.7' $\times 3.3^{\prime}$; Condon et al. 1989). By comparing W3 and HB3 at different radio frequencies, a clear boundary can be drawn. The thermal emission from W3 appears in similar regions at all frequencies and with no obvious decrease in strength, while the non-thermal emission from HB3 becomes progressively less prominent as the frequency increases. Because there is no apparent brightening of the SNR along the border with W3, this strongly supports Landecker et al.'s (1987) a conclusion that there is no interaction between HB3 and W3. Routledge et al.'s (1991) CO spectra observation reveals that HB3 has an interaction with molecular gas in which W3 is embedded, but no evidence for a direct interaction of W3 and HB3. Koralesky et al.'s (1998) maser study in the W3/HB3 complex also shows no evidence for any interaction between $\mathrm{HB} 3$ and $\mathrm{W} 3$.

Landecker et al.'s (1987) analysis on the uncertainty of the flux density of HB3 is consistent with our work, i.e., the uncertainty arises more from the difficulty of determining the background emission level than from imprecise separation of SNR from W3. We take two measures in order to calculate the integrated flux density of HB3. Firstly, we divide HB3 nonthermal region into 5 subareas (see the division in Table 3). We remove all strong compact sources within HB3 and individually integrate all strong compact sources (integrated flux density is $5.3 \pm 0.4 \mathrm{Jy}$ ). After subtracting an average background emission value, we calculate an average pure emission value in each subarea, and then substitute the average value for the areas removed due to compact sources. We then integrate five subareas and get the first estimate value of HB3 flux density $(68.6 \pm 11.5 \mathrm{Jy})$. As a comparison procedure, we do the same removal of the compact sources, then we calculate a separate background value for each subarea and substitute the resulting subarea average emission value for areas removed due to compact sources. Then we sum the integrated flux values for the five subareas to get the second estimated value of integrated flux density $(72.9 \pm 9.9 \mathrm{Jy})$. Our results are consistent with each other and also with Landecker et al.

Fesen et al. (1995) observed HB3 at both $408 \mathrm{MHz}$ and $1420 \mathrm{MHz}$ with the DRAO ST and estimated its integrated flux density $(48.3 \pm 6.5 \mathrm{Jy}$ at $408 \mathrm{MHz}, 26.0 \pm 2.7 \mathrm{Jy}$ at $1420 \mathrm{MHz})$, and calculated the spectrum index $(0.5 \pm 0.2)$. The flux density they gave is rather low in comparison to previous authors' and ours. Their explanation that they subtracted the strong compact sources before integrating is not convincing because the total flux density of these compact sources we find is only $7 \%$ at $408 \mathrm{MHz}$ and $5 \%$ at $1420 \mathrm{MHz}$ of HB3's flux density. Fesen et al.'s (1995) calculation probably excluded too much area near the HB3-W3 boundary. They obtained a spectral index value $(0.64 \pm 0.01)$ using the $\mathrm{T}-\mathrm{T}$ plot method, but there is no description of the selected area. It is possible to get a steep spectral index from one area (e.g., subbox $2 \mathrm{c}$ or $6 \mathrm{~d}$ ) according to our results, but the average value from the whole area of HB3 is much less than 0.6.

Reich et al. (2003) executed their observations of HB3 at $35 \mathrm{~cm}$ wavelength with the Effelsberg 100-m radio telescope, and reported an integrated flux density of $51.5 \pm 3.5 \mathrm{Jy}$. There is no information concerning their estimation method except they mentioned the difficulty to obtain accurate flux density of HB3. Trushkin (2002) predicts about $57.8 \mathrm{Jy}$ at $863 \mathrm{MHz}$ based on all available data until 2002 and calculated a spectral index of 0.49 .

\subsection{Explanation of HB3 spectral index variation}

We have seen a gradient of thermal background emission from W3 decreasing toward the upper right in Fig. 1. By choice of background for the integrated flux measurement this smooth gradient is subtracted from the fluxes and spectral index. Similarly, by choosing small boxes for $\mathrm{T}-\mathrm{T}$ plots, we make the T-T plot spectral index much less sensitive to a large scale background gradient. An important point is that the spectral index of 0.32 obtained by fitting the higher frequency integrated flux data is consistent with the $408-1420 \mathrm{MHz}$ integrated flux density based spectral index $0.34 \pm 0.15$ and the best $\mathrm{T}-\mathrm{T}$ plot spectral index 0.36 .

Now we discuss other properties of the spectral index variations, both the variation with frequency (Fig. 3) and 
the variation of $408-1420 \mathrm{MHz}$ spectral index with location within HB3 (Tables 1, 2 and 3). Since the integrated flux and $\mathrm{T}-\mathrm{T}$ plot derived spectral indices agree, the filamentary emission (measured by the T-T plot method) and the total emission (filamentary plus spatially smooth emission) have the same spectral index. This agreement between $\mathrm{T}-\mathrm{T}$ plot and integrated flux spectral index holds for the whole of HB3 and also for the five subregions given in Table 3 . When we consider the $\mathrm{T}-\mathrm{T}$ plot results in the finest detail (Table 2), we find a range of spectral indices, generally in the range 0.1 to 0.7 (with errors $0.1-0.2$ ). The set of $408-1420 \mathrm{MHz}$ spectral indices corresponds to a set of populations of electrons with different energy indices. Under the simplest assumption, each population has a power-law energy spectrum over a wide range of energies, so each population emits synchrotron radiation over a wide range of radio frequencies with a single spectral index. Then the summed radio spectrum would have a larger contribution from the steeper index electron populations at low frequencies, and a larger contribution from the flatter index electron populations at high frequencies. This is consistent with what is observed (Fig. 3). Alternatives, such as having electron populations with curved energy spectra in the normal sense with steeper energy index at higher energies, do not work since they would produce a curved radio spectrum with steeper spectral index at higher frequency, contrary to what is observed.

\section{Conclusion}

Our $408 \mathrm{MHz}$ and $1420 \mathrm{MHz}$ maps of the SNR HB3 reveal similar but more detailed weak emission structure features than previously published maps. Considering a background emission gradient across HB3 area, we give HB3's flux density at $408 \mathrm{MHz}, 1420 \mathrm{MHz}$ and $2695 \mathrm{MHz}$. The integrated flux-density based spectral index between $408 \mathrm{MHz}$ and $1420 \mathrm{MHz}$ is $0.34 \pm 0.15$. The averaged T-T plot spectral index is 0.36 . Based on the analysis of our data and previous data, we reconcile our spectral index result with the previous authors' 0.6 . A viable explanation is that on small spatial scales the spectral index varies within the range $0.1-0.7$. Thus the low frequency data mainly reflect the steeper emission components and the high frequency data mainly reflects the flatter emission components.

Acknowledgements. We thank the referee for his constructive suggestions which helped to improve the paper. Dr. R. Kothes at the DRAO provided information on calibration errors of the CGPS data. W.W. Tian thanks the MoST for grant NKBRSF 2003 CB716703 and NSF grant in China. We acknowledge support from the Natural Sciences and Engineering Research Council of Canada. The DRAO is operated as a national facility by the National Research Council of Canada. The Canadian Galactic Plane Survey is a Canadian project with international partners.

\section{References}

Caswell, J. L. 1967, MNRAS, 136, 11

Condon, J. J., Broderick, J. J., \& Seielstad, G. A. 1989, AJ, 97, 1064

Fesen, R. A., Downes, R. A., Wallace, D., et al. 1995, AJ, 110, 2876

Fürst, E., Reich, W., Reich, P., \& Reif, K. 1990, A\&AS, 85, 691

Haslam, C. G. T., Salter, C. J., Stoffel, H., \& Wilson, W. W. 1982, A\&AS, 47, 1

Kirralesky, B., Frail, D. A., Goss, W. M., et al. 1998, AJ, 116, 1323

Kovalenko, A. V., Pynzar, A. V., \& Udal'tsov, V. A. 1994 AR, 38, 95

Leahy, D. A., \& Roger, R. S. 1998, ApJ, 505, 784

Leahy, D. A., Venkatesan D., Long, K. S., \& Naranan, S. 1985, ApJ, 294, 183

Landecker, T. L, Vaneldik, J. F., Dewdney, P. E., et al. 1987, AJ, 94, 111

Reich, W., Reich, P., \& Fürst, E. 1990, A\&AS, 83, 539

Reich, W., Reich, P., \& Fürst, E. 1997, A\&AS, 126, 413

Reich, W., Zhang, X., \& Fürst, E. , 2003, A\&A, 408961

Rengelink, R. B., Tang, Y., de Bruyn, A. G., et al. 1997, A\&AS, 124, 259

Routledge, D., Dewdney, P. E., Landecker, T. L., et al. 1991, A\&A, 247,529

Taylor, A. R., Gibson, S. J., \& Peracaula, M. 2003, AJ, 125, 3145

Trushkin, S. A. 2002, http://cats.sao.ru

Trushkin, S. A. 1987, AISAO, 25, 84

Velusamy, T., \& Kundu, M. R. 1974, A\&A, 32, 375

Zhang, X. Z., Zheng, Y., Landecker, T. L., et al. 1997, A\&A, 324, 641

Zhu, J. 1993, in Master thesis, Radio Observation at $232 \mathrm{MHz}$ of Large Angular Supernova Remnant HB21 and HB3, Issued by Beijing Astronomical Observatory 